\title{
Apoptotic Markers Are Increased in Epilepsy Patients: A Relation with Manganese Superoxide Dismutase Ala16Val Polymorphism and Seizure Type through IL-1 $\beta$ and IL-6 Pathways
}

\author{
Aline Kegler, ${ }^{1,2}$ Ana Letícia Fornari Caprara, ${ }^{1}$ Eduardo Tanuri Pascotini, ${ }^{1,3}$ Josi Arend, $^{3}$ \\ Patricia Gabbi, ${ }^{3}$ Marta M. M. F. Duarte, ${ }^{3}$ Ana Flavia Furian, ${ }^{3}$ Mauro Schneider Oliveira, ${ }^{3}$ \\ Luiz Fernando Freire Royes, ${ }^{2,3,4}$ and Michele Rechia Fighera $\mathbb{D}^{1,2,3,4}$ \\ ${ }^{1}$ Centro de Ciências da Saúde, Departamento de Neuropsiquiatria, Universidade Federal de Santa Maria, RS, Brazil \\ ${ }^{2}$ Centro de Ciências Naturais e Exatas, Programa de Pós-Graduação em Ciências Biológicas: Bioquímica Toxicológica, \\ Universidade Federal de Santa Maria, RS, Brazil \\ ${ }^{3}$ Centro de Ciências da Saúde, Programa de Pós-Graduação em Farmacologia, Universidade Federal de Santa Maria, RS, Brazil \\ ${ }^{4}$ Centro de Educação Física e Desportos, Laboratório de Bioquímica do Exercício (BIOEX), Universidade Federal de Santa Maria, \\ RS, Brazil
}

Correspondence should be addressed to Michele Rechia Fighera; mrfighera@yahoo.com.br

Received 20 September 2019; Revised 11 February 2020; Accepted 26 February 2020; Published 6 March 2020

Academic Editor: Graciela Russomando

Copyright (c) 2020 Aline Kegler et al. This is an open access article distributed under the Creative Commons Attribution License, which permits unrestricted use, distribution, and reproduction in any medium, provided the original work is properly cited.

\begin{abstract}
The MnSOD Ala16Val single nucleotide polymorphism (SNP) has been associated with different diseases. However, there are scarcely studies relating this SNP in epilepsy, a neurologic disease that involves some interacting pathways, such as apoptotic and inflammatory factors. In this sense, we decided to investigate the relationship of MnSOD Ala16Val SNP with apoptotic markers in epilepsy and its relation with inflammatory pathway and seizure type. Ninety subjects were evaluated (47 epilepsies; 43 controls) by questionnaires and laboratorial exams. We observed a higher percentage of VV genotype in the epilepsy group when compared to the control group. IL-1 $\beta$, IL-6, caspase-1, and caspase-3 levels were increased in the epilepsy group (VV genotype). Furthermore, an important correlation between IL-1 $\beta$ vs. caspase- 1 and IL- 6 vs. caspase- 3 was observed in the epilepsy group (VV genotype). The epilepsy group which presented generalized seizures also demonstrated a positive correlation between IL-1 $\beta$ vs. CASP1 and IL-6 vs. CASP3. Thus, it is a plausible propose that epilepsy patients with VV genotype and generalized seizures present a worse inflammatory and apoptotic status. Our findings suggest that the knowledge of MnSOD Ala16Val polymorphism existence is important to evaluate molecular mechanisms associated to seizure and improve the treatment of these patients.
\end{abstract}

\section{Introduction}

Epilepsy is one of the most common neurological disorders [1] characterized by an enduring predisposition to generate seizures [2] affecting more than 65 million people worldwide [3]. Despite progress in pharmacological and surgical treatments of epilepsy, it is not clear about the processes leading to the generation of seizures and about the mechanisms; whereby, a healthy brain is rendered epileptic [4]. Apoptosis [5], neuroinflammation [4], and oxidative stress [6] are some relevant factors implicated in epilepsy pathophysiology. Many works have acknowledged the role of neuroinflammation in the pathogenesis of seizures, but little is known about the mechanisms that start the inflammatory 
process in epilepsy [7]. The microglia constitute the primary CNS immune cells and are quickly activated in response to an insult. However, the excessive activation of microglia may be harmful, promoting the development of neuronal diseases by producing large amounts of inflammatory molecules, such as IL-6 [8], IL- $1 \beta$, and reactive oxygen species (ROS) [9]. In epilepsy, there is a complex cascade of molecular and cell mechanisms involved in excitotoxicity [10], oxidative stress [11], and inflammation [4] beyond cytotoxicity mediated by cytokines [8] and cell death pathway activation [12]. In fact, when the brain is affected by brain diseases (i.e., epilepsy), the microglia cells are activated [13], and this activation may lead to production of inflammatory cytokines as IL- $\beta$ [14] and IL-6 [15]. Interestingly, some antioxidant molecules were reported to decrease the levels of proinflammatory mediators by scavenging ROS [16]. Therefore, the redox balance is thought to regulate a series of neuroinflammatory processes mediated by microglia [9]. Manganese superoxide dismutase (MnSOD) antioxidant enzyme is the only known major defense against reactive oxygen species within mitochondria [17]. Furthermore, MnSOD is reportedly induced in the CNS under inflammatory conditions [9]. Regarding the relevance of MnSOD, numerous factors can impact on the effectiveness of antioxidant enzymes, including enzymatic polymorphism [18]. Two main MnSOD SNPs have been described in the literature, one of which is Ala16Val [17]. The change of alanine (Ala) to valine (Val) at the $16^{\text {th }}$ amino acid (Ala16Val) of the signal sequence of MnSOD is suggested to change the structure of the protein. The alanine-to-valine substitution produces a $\beta$-sheet secondary structure instead of an $\alpha$-helix structure, decreasing the enzyme transport efficiency into the mitochondria and compromising the antioxidant potential [19]. The Ala16Val MnSOD SNPs generate three possible genotypes: AA, AV, and VV. Sutton et al. [20] reported that the Val allele results in reduced expression and production of an unstable mRNA, affecting the import of SOD2 into the mitochondria. Accordingly, Montano et al. [21] demonstrated that the VV and AV peripheral blood mononuclear cells (PBMC) presented increased levels of inflammatory cytokines as IL- $1 \beta$ and IL- 6 .

There are studies reporting the association of inflammatory and apoptotic parameters with MnSOD Ala16Val SNP in diseases as stroke [22, 23] and cancer [24]. However, there are few evidences of involvement of Ala16Val MnSOD SNP in epilepsy [25]. In this sense, the aim of this study was to investigate caspases' activation and relationship between Ala16Val MnSOD SNP with interleukins in epilepsy patients. Additionally, we have also investigated the relation of seizure type (partial or generalized) and duration time (minutes) of seizures with parameters aforementioned. This study is of such interest in view that an enzymatic polymorphism has an important role in inflammatory and apoptotic pathway, and the obtained results can contribute to a better understanding about the epilepsy disease and its mechanisms, maybe providing support to novel approaches of pharmacotherapy.

\section{Materials and Methods}

\subsection{Study Design}

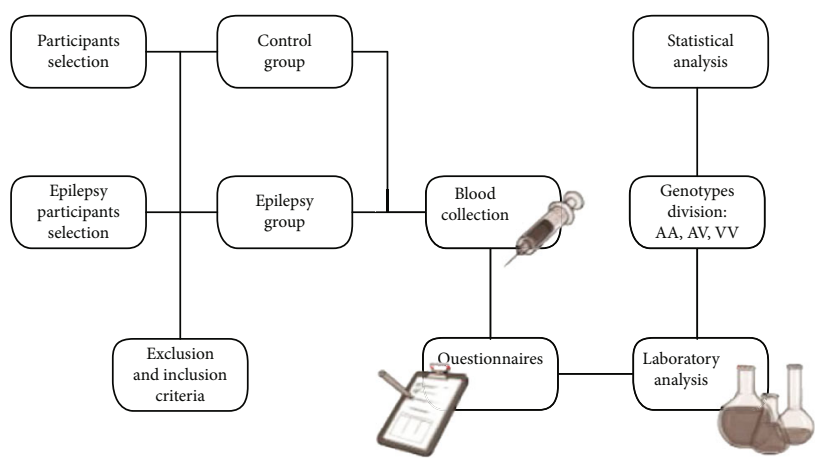

2.2. Participants. We performed a case-control study and a total of 90 subjects were recruited and allocated into two groups: epilepsy group $(n=47)$ and control group (healthy subjects, $n=43$ ). No etiology was found after detailed history, physical, laboratory, and imaging studies. Major exclusion criteria were history of autoimmune, liver, kidney, and inflammatory diseases; allergic response; immune deficiency disorder; diabetes, psychiatric illness; malignancy; smoking; or a systemic or central nervous system (CNS) infection 2 weeks before sample collection. The study protocol was approved by the local institutional review boards at the authors' affiliated institutions. Informed written consent was obtained from all the subjects or their legal surrogates. The work described has been carried out in accordance with The Code of Ethics of the World Medical Association (Declaration of Helsinki).

2.3. Epilepsy Group. The epilepsy patients were recruited from the University Hospital of Santa Maria and invited to volunteer for the study. Epilepsy was diagnosed by two experienced neurologists according to the 2010 International League Against Epilepsy (ILAE) Classification, revised in 2017 ([26]; Fisher et al., 2017; Scheffer et al., 2017). All patients were evaluated for seizure frequency using seizure diaries [27]. The seizure type from epilepsy group $(n=47)$ was confirmed through interviews with the patients and their relatives as well as EEG analysis and tomography or magnetic resonance imaging (MRI). For a diagnosis of generalized epilepsy, the patient would typically show generalized spikewave activity on EEG. Individuals with generalized epilepsies may have a range of seizure types including absence, myoclonic, atonic, tonic, and tonic-clonic seizures. The diagnosis of generalized epilepsy is made on clinical grounds, supported by the finding of typical interictal EEG discharges. Caution needs to be exercised for a patient with generalized tonic-clonic seizures and a normal EEG. In this case, supportive evidence would need to be presented to make a diagnosis of generalized epilepsy, such as myoclonic jerks or a relevant family history. Focal epilepsies include unifocal and multifocal disorders as well as seizures involving one hemisphere. A range of seizure types can be seen including focal aware seizures, focal impaired awareness seizures, focal motor seizures, focal nonmotor seizures, and focal to bilateral 
tonic-clonic seizures. The interictal EEG typically shows focal epileptiform discharges, but the diagnosis is made on clinical grounds, supported by EEG findings. The term "unknown" is used to denote where it is understood that the patient has epilepsy, but the clinician is unable to determine if the epilepsy type is focal or generalized because there is insufficient information available.

In our study, forty-five patients were in remission except for two patients who were diagnosed with refractory epilepsy. All epilepsy patients had normal neurological examinations except for one who presented tetra paresis secondary to spinal cord lesion. All epilepsy had normal 1.5 T MRI; one patient had right and left hippocampal sclerosis.

\subsection{Study Variables. Sex (dichotomous): male and female Age (quantitative): years \\ Antiepileptic drugs (quantitative): number of drugs used for each patient \\ MnSOD Ala16Val genotype AA, AV, and VV (quantita- tive): frequencies (\%) \\ Epilepsy type (dichotomous): generalized, focal, and unknown \\ Protein carbonyl (quantitative): $\mathrm{nmol} / \mathrm{mg}$ protein \\ SOD2 activity (quantitative): $\mathrm{U} / \mathrm{mg}$ hemoglobin \\ IL-1 $\beta$ (quantitative): $\mathrm{pg} / \mathrm{mL}$ \\ IL-6 (quantitative): (pg/mL) \\ Caspase-3 (quantitative): $\mathrm{mg} / \mathrm{mL}$ \\ Caspase-1 (quantitative): $\mathrm{mg} / \mathrm{mL}$}

2.5. Laboratory Analyses. Samples were collected at least 7 days from the last seizure attack (Mao et al., 2013). After $12 \mathrm{~h}$ of overnight fasting, blood samples were collected by venipuncture using purple, green, and red top Vacutainer ${ }^{\circledR}$ (BD Diagnostics, Plymouth, UK) tubes with ethylenediamine tetra acetic acid (EDTA), heparin, or no anticoagulants, respectively. The specimens were routinely centrifuged within $1 \mathrm{~h}$ of collection for $15 \mathrm{~min}$ at $2500 \mathrm{~g}$ and aliquots of the serum samples, and the supernatant was saved and stored at $-80^{\circ} \mathrm{C}$ for subsequent laboratory analysis, according to specific methods.

2.6. Protein Carbonyl (PC). The analysis of protein carbonyl was in accordance with [28].

2.7. Manganese Superoxide Dismutase (MnSOD). The manganese superoxide dismutase activity was performed in accordance with [29].

2.8. Caspase Determination. Caspase- 1 and caspase- 3 activities were determined by Fluorimetric Assay Kits (BioVision, Mountain View, CA). The fluorescence intensity was recorded at wavelength of $400 \mathrm{~nm}$ for excitation and at wavelength of $505 \mathrm{~nm}$ for emission for both. The activity was then calculated as fluorescence intensity $(\mathrm{FI}) / \mathrm{min} / \mathrm{mL}=$ $\Delta \mathrm{Flt} /(\mathrm{t} \times \mathrm{v})$, where $\Delta \mathrm{Flt}$ is the difference in fluorescence intensity between time zero and time $t$ minutes, tis the reaction time in min, and vis the volume of sample in $\mathrm{mL}$.
2.9. Cytokine Determination. The cytokines were assessed by ELISA using commercial kits for human IL- $\beta$ and IL-6 (eBioscience, San Diego, USA).

2.10. DNA Damage. The alkaline DNA comet assay as described by Pereira. Genomic DNA was isolated from peripheral blood leukocytes using a DNA Mini Kit Purification (Mo Bio).

2.11. MnSOD Ala16Val Genotyping. Genomic DNA was isolated from peripheral blood leukocytes using a DNA Mini Kit Purification (Mo Bio). MnSOD Ala16Val SNP was detected by PCR-RFLP according to Taufer et al. PCR amplifications were performed in a total volume of $50 \mu \mathrm{l}$ containing $5 \mu \mathrm{l}$ of $10 \mathrm{x}$ buffer, $1 \mu \mathrm{l}$ of $25 \mathrm{mM} \mathrm{MgCl}$, $1.25 \mu \mathrm{l}$ of $10 \mathrm{mM}$ dNTP, $0.5 \mu \mathrm{l}$ of Taq polymerase (Gibco Inc., Co.), $1 \mu \mathrm{l}$ of each primer ( $40 \mathrm{pmol}), 3 \mu \mathrm{l}$ of genomic DNA $(0.25 \mu \mathrm{g})$, and $34.5 \mu \mathrm{l}$ of ddH2O. The amplification primers (Gibco Inc., Co.) for a $110 \mathrm{bp}$ fragment of the human MnSOD gene were $5^{\prime}$-ACCAGCAGGCAGCTGG CGCCGG-3' (sense strand) and $5^{\prime}$-GCGTTGATGTG AGGTTCCAG-3' (antisense strand) with the following thermocycler parameters: an initial cycle of $95^{\circ} \mathrm{C}$ for $5 \mathrm{~min}$ followed by 35 cycles at $95^{\circ} \mathrm{C}$ for $1 \mathrm{~min}$ and $61^{\circ} \mathrm{C}$ for $1 \mathrm{~min}$. The final cycle was followed by an extension period of $2 \mathrm{~min}$ at $72^{\circ} \mathrm{C}$. The PCR product $(10 \mu \mathrm{l})$ was digested with Hae III ( $15 \mathrm{U} ; 37^{\circ} \mathrm{C}$; $6 \mathrm{~h}$; Gibco Inc., Co.). Digested products ( 23 and $85 \mathrm{bp}$ ) were visualized on a $4 \%$ agarose gel (Amersham Biosciences Inc., Co.) stained with ethidium bromide. A mutation was introduced by a primer mismatch to create a restriction cut site for Hae III in the -9 codon, and the following genotypes were observed: -9Ala/Ala (23 and $85 \mathrm{bp}) ;-9 \mathrm{Ala} / \mathrm{Val}(23,85$, and $110 \mathrm{bp})$; and $-9 \mathrm{Val} / \mathrm{Val}$ (110 bp).

2.12. Sample Size. As there is no comparison in the literature of apoptotic, inflammatory, and oxidative levels with MnSOD polymorphism in epilepsy patients, an adequate calculation if the sample size is not possible. Considering a significant difference of a standard deviation between the two groups and using the PEPI software, considering a study power of $90 \%$ and an alpha error of $0.05,46$ patients would be needed.

2.13. Statistical Methods. Data were analyzed by analysis of variance (two-way ANOVA) followed by Tukey's multiple comparison test. Statistical analysis was performed using the SPSS (Statistical Package for the Social Sciences) software in a PC-compatible computer. Correlation analyses were carried out using the Pearson correlation coefficient. Statistical significance was assumed when $p<0.05$. Chi-square test was used to calculate sex, age, and genotype frequencies.

\section{Results}

Baseline characteristics of the participants are described in Table 1. According to Chi-square analysis, no statistically difference was observed between the epilepsy group and control group relating with sex $(p=0.5)$ and age $(p=0.6)$. 
TABLE 1: Characteristics of epilepsy and control groups.

\begin{tabular}{lccc}
\hline Characteristics & $\begin{array}{c}\text { Epilepsy } \\
(n=47)\end{array}$ & $\begin{array}{c}\text { Control } \\
(n=43)\end{array}$ & $p$ value \\
\hline Gender & & & \\
$\quad$ Male & $22(46,8 \%)$ & $23(53,4 \%)$ & 0.5 \\
$\quad$ Female & $25(53,1 \%)$ & $20(46,5 \%)$ & \\
Mean age (years old) & & & \\
$\quad$ Male & 36 & 39 & 0.6 \\
$\quad$ Female & 33 & 42 & \\
Antiepileptic drugs (AEDs) & & & \\
$\quad \begin{array}{l}\text { Monotherapy } \\
\text { Polytherapy }\end{array}$ & $32(31,9 \%)$ & & \\
\hline
\end{tabular}

Analysis of the Ala16Val MnSOD gene yielded three variants of the genotype: AA (wild type), AV (heterozygous), and VV (homozygous). The Ala16Val MnSOD genotype frequencies were calculated and are presented in Table 2. In the epilepsy group, the genotype frequencies were $31.9 \%$ for AA, $21.2 \%$ for $\mathrm{AV}$, and $46.8 \%$ for $\mathrm{VV}$. The frequencies for AA, $\mathrm{AV}$, and $\mathrm{VV}$ genotypes were $39.5 \%, 32.5 \%$, and $27.9 \%$, respectively, in the control group. According to Chi-square analysis, no statistically difference in Ala16Val MnSOD genotype frequencies was observed $(p=0.1)$.

3.1. Protein Carbonyl (PC). A two-way ANOVA demonstrated increased protein carbonyl levels in the epilepsy group when compared to the control group $(F(1,84)=36.48$, $p<0.0001)$. Post hoc analysis with Tukey's test for multiple comparisons revealed increased levels of PC in the epilepsy group (AA, AV, and VV genotypes) when compared to their genotypes from the control group, respectively (Figure 1).

3.2. Manganese Superoxide Dismutase (MnSOD). A two-way ANOVA demonstrated increased MnSOD enzyme activity in the epilepsy group when compared to the control group $(F(1,81)=617.5, p<0.0001)$. Post hoc analysis with Tukey's test for multiple comparisons revealed increased MnSOD activity in the epilepsy group (AA, AV, and VV genotypes) when compared to their genotypes from the control group, respectively. Furthermore, in the epilepsy group, the homozygous VV genotype presented decreased enzyme activity when compared to AA genotype (Figure 2).

3.3. IL-1 $\beta$. A two-way ANOVA demonstrated increased IL$1 \beta$ levels in the epilepsy group when compared to the control group $(F(2,85)=6.2, p<0.01)$. Post hoc analysis with Tukey's test for multiple comparisons revealed increased IL- $1 \beta$ levels in the epilepsy group (AA, AV, and VV genotypes) when compared to their genotypes from the control group, respectively. Furthermore, in the epilepsy group, the homozygous VV genotype presented increased levels when compared to AV and AA genotypes (Figure 3).

3.4. IL-6. A two-way ANOVA demonstrated increased IL-6 levels in the epilepsy group when compared to the control group $(F(2,77)=4.6, p<0.05)$. Post hoc analysis with Tukey's test for multiple comparisons revealed increased
TABLE 2: MnSOD Ala16Val genotype frequencies in epilepsy and control groups.

\begin{tabular}{lccc}
\hline MnSOD SNP & Epilepsy & Control & $p$ value \\
\hline Genotypes & & & \\
AA & $15(31.9 \%)$ & $17(39.5 \%)$ & \\
AV & $10(21.2 \%)$ & $14(32.5 \%)$ & 0.1 \\
VV & $22(46.8 \%)$ & $12(27.9 \%)$ & \\
\hline
\end{tabular}

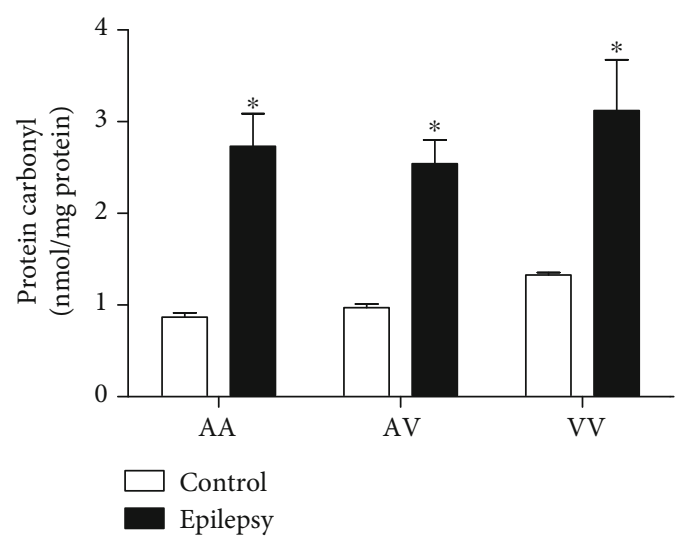

FIgURE 1: Comparison of Ala16Val MnSOD polymorphism genotypes (AA, AV, and VV) from control and epilepsy groups in relation to oxidative stress biomarker. The epilepsy group presented increased levels of protein carbonyl when compared to control group. ${ }^{*} p<0.05$ when compared to respective control group.

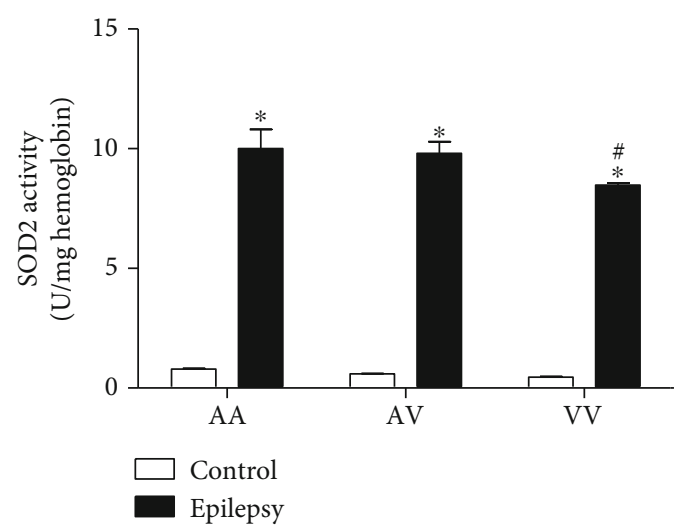

FIgURE 2: Comparison of Ala16Val MnSOD polymorphism genotypes (AA, AV, and VV) from control and epilepsy groups in relation to SOD2 activity. The epilepsy group presented increased SOD2 activity when compared to the respective control group. The epilepsy group (VV) presented a decreased SOD2 activity when compared to epilepsy group (AA). ${ }^{*} p<0.05$ when compared to respective control group; $\# p<0.05$ when compared to the epilepsy group (VV vs. AA).

IL-6 levels in the epilepsy group (AA, AV, and VV genotypes) when compared to their genotypes from the control group, respectively. Furthermore, in the epilepsy group, the homozygous VV genotype presented increased levels when compared to AA genotype (Figure 4). 


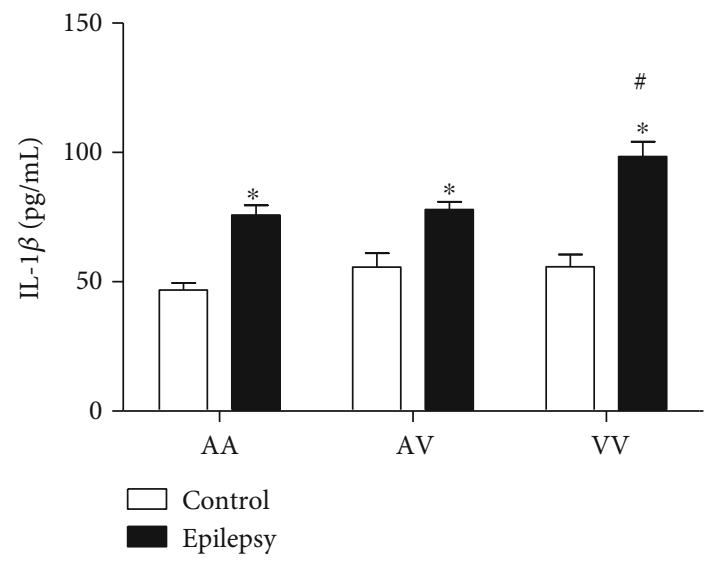

Figure 3: Comparison of Ala16Val MnSOD polymorphism genotypes (AA, AV, and VV) from control and epilepsy groups in relation to IL-1 $\beta$. The epilepsy group presented increased levels of IL- $1 \beta$ when compared to control group. ${ }^{*} p<0.05$ when compared to respective control group; ${ }^{\#} p<0.05$ when compared to the epilepsy group (VV vs. AV and AA).

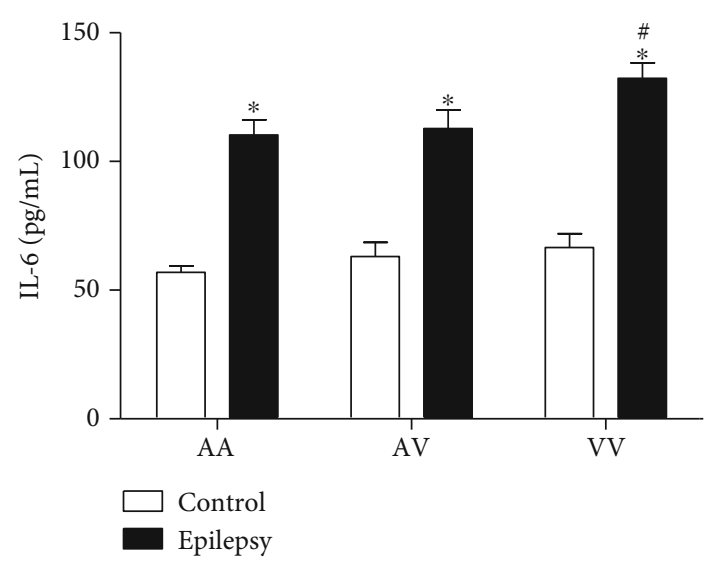

FIgUre 4: Comparison of Ala16Val MnSOD polymorphism genotypes (AA, AV, and VV) from control and epilepsy groups in relation to IL-6. The epilepsy group presented increased levels of IL-6 when compared to control group. ${ }^{*} p<0.05$ when compared to respective control group; ${ }^{\#} p<0.05$ when compared to the epilepsy group (VV vs. AA).

3.5. Caspase-3. A two-way ANOVA demonstrated increased caspase-3 levels in the epilepsy group when compared to the control group $(F(2,77)=6.9, p<0.01)$. Post hoc analysis with Tukey's test for multiple comparisons revealed increased caspase-3 levels in the epilepsy group (AA, AV, and VV genotypes) when compared to their genotypes from the control group, respectively. Furthermore, in the epilepsy group, the homozygous VV genotype presented increased levels when compared to AA genotype (Figure 5).

3.6. Caspase-1. A two-way ANOVA demonstrated increased caspase-1 levels in the epilepsy group when compared to the control group $(F(2,77)=3.8, p<0.05)$. Post hoc analysis with Tukey's test for multiple comparisons revealed increased caspase-1 levels in the epilepsy group (AA, AV, and $\mathrm{VV}$ genotypes) when compared to their genotypes from

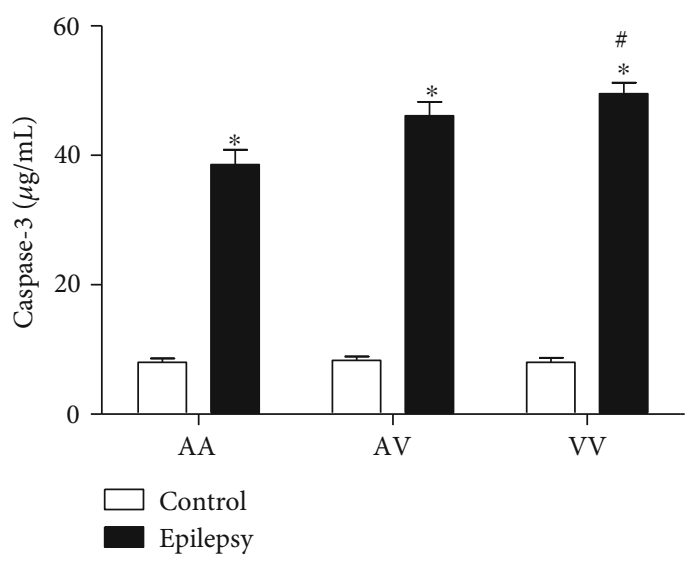

FIgURE 5: Comparison of Ala16Val MnSOD polymorphism genotypes (AA, $\mathrm{AV}$, and $\mathrm{VV}$ ) from control and epilepsy groups in relation to caspase-3. The epilepsy group presented increased levels of caspase-3 when compared to control group. ${ }^{*} p<0.05$ when compared to respective control group; ${ }^{*} p<0.05$ when compared to the epilepsy group (VV vs. AA).

the control group, respectively. Furthermore, in the epilepsy group, the homozygous $\mathrm{VV}$ genotype presented increased levels when compared to AA genotype (Figure 6).

3.7. Comet Assay. A two-way ANOVA demonstrated increased amount of DNA damage in the epilepsy group when compared to the control group $(F(1,84)=1282, p<$ $0.0001)$. Post hoc analysis with Tukey's test for multiple comparisons revealed increased amount of DNA damage in epilepsy group (AA, AV, and VV genotypes) when compared to their genotypes from the control group, respectively (data not shown).

\subsection{Correlations}

3.8.1. IL-1 $\beta$ vs. Caspase-1. Pearson's analysis demonstrated an interesting correlation between IL- $1 \beta$ and caspase- 1 $(r=0.7, p<0.001)$ in the epilepsy group (VV genotype) (Table 3).

3.8.2. IL-6 vs. Caspase-3. Pearson's analysis demonstrated an interesting correlation between IL- 6 and caspase- $3(r=0.5$, $p<0.05)$ in the epilepsy group (VV genotype) (Table 3 ).

3.8.3. Seizure Type vs. Polymorphism. Pearson's analysis demonstrated in the epilepsy group which presented generalized seizures (VV genotype), an interesting correlation between inflammatory and apoptotic parameters: IL- $1 \beta$ vs. caspase$1(r=0.7, p<0.05)$ and IL-6 vs. caspase-3 $(r=0.6, p<0.05)$. Furthermore, the results demonstrated an increased in caspase-1 levels in the epilepsy group which presented generalized seizures (VV genotype) $(t=2.89, p<0.05)$. The other parameters did not demonstrate significant alteration in relation to generalized or partial seizures (data not shown) (Table 3).

3.8.4. Seizures' Duration Time (Minutes). The statistical analysis revealed that the epilepsy group which presented generalized seizures (VV genotype) presented longer seizure time 


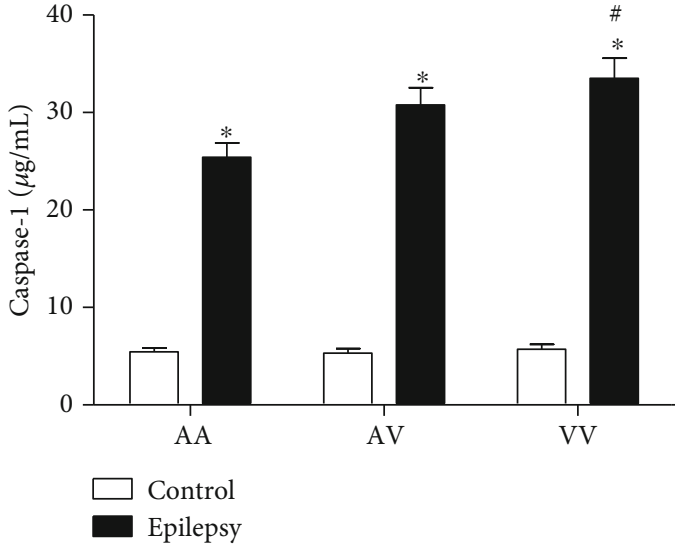

Figure 6: Comparison of Ala16Val MnSOD polymorphism genotypes (AA, AV, and VV) from control and epilepsy groups in relation to caspase-1. The epilepsy group presented increased levels of caspase-1 when compared to control group. ${ }^{*} p<0.05$ when compared to respective control group; ${ }^{\#} p<0.05$ when compared to the epilepsy group (VV vs. AA).

TABLE 3: Correlation among inflammatory, apoptotic, and DNA damage parameters with VV genotype in the epilepsy group and epilepsy group with generalized seizures.

\begin{tabular}{lcc}
\hline Correlation & $r$ value & $p$ value \\
\hline VV genotype-epilepsy group & & \\
IL-1 $\beta$ vs. caspase-1 & 0.7 & $<0.001$ \\
IL-6 vs. caspase-3 & 0.5 & $<0.05$ \\
VV genotype-epilepsy group (generalized seizures) & \\
IL-1 $\beta$ vs. caspase-1 & 0.7 & $<0.05$ \\
IL-6 vs. caspase-3 & 0.6 & $<0.05$ \\
\hline
\end{tabular}

(minutes) than the epilepsy group which presented partial seizures (VV genotype) $(t=2.46, p<0.05)$.

\section{Discussion}

The novel finding of the study is the influence of Ala16Val MnSOD gene polymorphism-VV genotype on inflammatory (IL-6, IL-1 $\beta$ ), apoptotic (caspases -1 and -3) and antioxidant enzyme (MnSOD) in epilepsy. Of such interest, we observed an interesting correlation (caspase-1 vs. IL-1 $\beta$ ) and (caspase-3 vs. IL-6) in VV epilepsy patients. Furthermore, the generalized seizures were impacted by the VV genotype in relation to the referred parameters and with relation to seizures' duration time. The burst firing neurons associated with epileptic discharges could lead to changes with events of cascades at the cellular level [30]. The complex mechanism of epileptogenesis remains largely unclear. However, oxidative stress by free radical generation does indeed play a role in mitochondrial dysfunction [31]. Furthermore, the oxidative stress can alter/influence factors leading to neuronal death, and, consequently the DNA damage [32]. The intense seizure activity can lead to cytotoxic effects mediated by oxidative stress. The superoxide anion $\left(\mathrm{O}_{2}^{-}\right)$is the central mediator of oxidative stress, influencing both physiological and pathological processes [33]. While there are some evidences confirming that oxidative stress manifest as a consequence of the first insult, which turns out later to become the cause of epileptogenesis [34], other studies support the influence of oxidative stress in epilepsy. In accordance with Patel [34], oxidative stress is the cause or consequence of epileptic seizures.

Protein oxidation is an irreversible oxidative damage, considered to be a marker for severe oxidative stress [28]. Our results demonstrated increased levels of protein carbonyl when compared with the epilepsy vs. control group. Accordingly, Sudha et al. [35] described protein carbonyl increased levels in epilepsy patients than in controls. Of such importance, when analyzing MnSOD, the results suggested that the polymorphism plays an influence on its performance, in view that the homozygous VV epilepsy group demonstrated decreased activity when compared to AV and AA genotypes.

The apoptotic pathway occurs primarily through the extrinsic and intrinsic pathways [36]. In relation to intrinsic (or mitochondrial) pathway, the apoptosis can be initiated by cytokines such as IL-6. In this pathway, the mitochondria release cytochrome $c$, activating the caspase-3, leading to cell death [37]. Of note, our results demonstrated increased IL-6 and caspase- 3 levels in the epilepsy group when compared to the control group. Accordingly, Peltola et al. [38] reported increased levels of IL-6 in plasma and cerebrospinal fluid (CSF) of epilepsy patients when compared to nonepilepsy patients. Increased caspase- 3 in brain tissues has been found in animal models of epilepsy [12] and epilepsy patients [39]. Studies also relate increased serum caspase- 3 with traumatic brain injury (TBI). However, caspase- 3 has been scarcely explored in blood of epilepsy patients [40]. The Ala16Val MnSOD polymorphism also revealed a significant importance when associated with the genotype: the VV epilepsy group demonstrated increased levels of IL- 6 and caspase-3 when compared to other genotypes ( $\mathrm{AV}$ and $\mathrm{AA}$ ). In this context, an interesting correlation between IL- 6 vs. caspase3 was observed. When compared to seizure type, a positive correlation was obtained when related to generalized seizures. Our results suggest that the Val allele has less efficiency, in view that the analyzed parameters were increased in the VV epilepsy group. Particularly, in the brain, these proteins can lead to the activation of caspase-3, inducing cell damage, such as DNA fragmentation [41]. In this study, we could also observe that in the epilepsy group, caspase- 1 and IL- $1 \beta$ presented increased levels when compared to the control group. Accordingly, Shi et al. [42] published a study relating that the cytokine IL- $1 \beta$ has also been found to be significantly increased within the CSF in epilepsy pediatric population when compared to control group, suggesting the cytokine's important role in epilepsy initiation and progression. Of such interest, in view that the IL- $1 \beta$ has proconvulsant actions, it is likely that this cytokine is released by cells following an inciting event. In this sense, a complex activation, which includes the caspase- 1 is a crucial step required for IL- $1 \beta$ release [43]. Accordingly, our results demonstrated an interesting correlation between IL- $1 \beta$ and caspase-1 in the epilepsy group-generalized seizures (VV 
genotype), corroborating with previous studies, emphasizing the great importance of Ala16Val MnSOD SNP seizure type in the obtained results. Still regarding to seizure type, it comes important in view that in generalized seizures, the disorders would be widespread throughout the brain [44]. When related to genetic polymorphism of MnSOD, we found some important results when analyzed AA, AV, and VV genotypes in the epilepsy group. It was observed a decreased MnSOD activity in the VV epilepsy group when compared to the AA epilepsy group. In this sense, our results are in accordance with that the ValVal could be less efficient than the AlaAla genotype to control the oxidative stress [17]. The V allele presents increased superoxide radical levels than the A allele due to its lower efficiency to dismutate this molecule into $\mathrm{H}_{2} \mathrm{O}_{2}$ [45]. Accordingly, the superoxide anion $\left(\mathrm{O}_{2}{ }^{-}\right)$is the central mediator of oxidative stress, this anion could lead to mitochondrial destabilization resulting in cell apoptosis activation [46]. Although the small sample size of the study, there are few studies indicating the association/influence of Ala16Val MnSOD polymorphism in epilepsy [25, 47]. We found some important associations with inflammatory, apoptotic, and oxidative stress biomarkers, suggesting that the Ala16Val MnSOD polymorphism has an important role on neuroinflammation maintenance and its consequences.

\section{Conclusion}

Finally, the result demonstrated influence of Ala16Val MnSOD polymorphism, mainly of VV genotype in epilepsy patients. Accordingly, studies have shown that the polymorphism of some genes may be related to the efficacy, tolerability, and action of antiepileptic drugs [48, 49].

In this sense, prolonged seizures or status epilepticus in epilepsy patients can become a serious problem due to their consequences on the quality life from this population [50]. Seizures can have devastating consequences and, as result, suffer bodily injury requiring hospitalization. Others have shortened life span due to the increased risk of unexpected sudden death that is associated with uncontrolled seizures [50-52]. Studies have shown that patients with epilepsy can be significant neuropsychological, psychiatric, and social impairments that limit employment, reduce marriage rates, and decrease quality of life [51-53]. Thus, genetic polymorphism becomes an "ally" to help discover the cause of drug refractoriness and provides insight into the type and magnitude of clinic-laboratorial manifestations may have across individuals, helping to determine the best treatment and improve the quality of life of patients with neurological diseases, such as epilepsy.

\section{Data Availability}

The data availability data used to support the findings of this study are included within the article.

\section{Ethical Approval}

The ethical approval code is CAAE 10554612.1. 0000.5346. The date of approval is November 20, 2012.

\section{Conflicts of Interest}

The authors declare there are any potential conflicts of interest. The authors declare they have no actual or potential competing financial interests

\section{Authors' Contributions}

All authors contributed equally in the study. All authors read and approved the final manuscript.

\section{Acknowledgments}

The work was supported by the CNPq (grant: 500120/2003-0). M.R. Fighera and L.F. F. Royes are the recipients of $\mathrm{CNPq}$ fellowships. A. Kegler and E. T. Pascotini are the recipients of CAPES fellowships.

\section{References}

[1] E. Perucca, J. French, and M. Bialer, "Development of new antiepileptic drugs: challenges, incentives, and recent advances," The Lancet Neurology, vol. 9, pp. 793-804, 2007.

[2] R. S. Fisher, W. van Emde Boas, W. Blume et al., "Epileptic seizures and epilepsy: definitions proposed by the International League Against Epilepsy (ILAE) and the International Bureau for Epilepsy (IBE)," Epilepsia, vol. 4, pp. 470-472, 2005.

[3] P. Kwan, S. C. Schachter, and M. J. Brodie, "Drug-resistant epilepsy," The New England Journal of Medicine, vol. 10, pp. 919-926, 2011.

[4] A. Vezzani, J. French, T. Bartfai, and T. Z. Baram, "The role of inflammation in epilepsy," Nature Reviews Neurology, vol. 1, pp. 31-40, 2011.

[5] A. K. Liou, R. S. Clark, D. C. Henshall, X. M. Yin, and J. Chen, "To die or not to die for neurons in ischemia, traumatic brain injury and epilepsy: a review on the stress-activated signaling pathways and apoptotic pathways," Progress in Neurobiology, vol. 69, no. 2, pp. 103-142, 2003.

[6] E.-J. Shin, J. H. Jeong, Y. H. Chung et al., "Role of oxidative stress in epileptic seizures," Neurochemistry International, vol. 2, pp. 122-137, 2011.

[7] A. C. da Fonseca, D. Matias, C. Garcia et al., "The impact of microglial activation on blood-brain barrier in brain diseases," Frontiers in Cellular Neuroscience, vol. 8, article 362, 2014.

[8] G. Li, S. Bauer, M. Nowak et al., "Cytokines and epilepsy," Seizure, vol. 3, pp. 249-256, 2011.

[9] Y. Ishihara, T. Takemoto, K. Itoh, A. Ishida, and T. Yamazaki, "Dual role of superoxide dismutase 2 induced in activated microglia: oxidative stress tolerance and convergence of inflammatory responses," Journal of Biological Chemistry, vol. 37, pp. 22805-22817, 2015.

[10] C. H. Cho, "New mechanism for glutamate hypothesis in epilepsy," Frontiers in Cellular Neuroscience, vol. 7, p. 127, 2013.

[11] J. N. Pearson-Smith and M. Patel, "Metabolic dysfunction and oxidative stress in epilepsy," International Journal of Molecular Sciences, vol. 18, no. 11, article 2365, 2017.

[12] D. C. Henshall and B. S. Meldrum, "Cell death and survival mechanisms after single and repeated brief seizures," in Jasper's Basic Mechanisms of the Epilepsies, NCBI Bookshelf, 4th edition, 2012. 
[13] K. Nakajima and S. Kohsaka, "Microglia: activation and their significance in the central nervous system," Journal of Biochemistry, vol. 130, no. 2, pp. 169-175, 2001.

[14] A. Vezzani and T. Z. Baram, "New roles for interleukin-1 beta in the mechanisms of epilepsy," Epilepsy Currents, vol. 2, pp. 45-50, 2007.

[15] A. Rana and A. E. Musto, "The role of inflammation in the development of epilepsy," Journal of Neuroinflammation, vol. 15, no. 1, article 144, 2018.

[16] J. P. Godbout, B. M. Berg, K. W. Kelley, and R. W. Johnson, " $\alpha$-Tocopherol reduces lipopolysaccharide-induced peroxide radical formation and interleukin-6 secretion in primary murine microglia and in brain," Journal of Neuroimmunology, vol. 149, no. 1-2, pp. 101-109, 2004.

[17] G. Bresciani, I. B. M. Cruz, J. A. de Paz, M. J. Cuevas, and J. González-Gallego, "The MnSOD Ala 16Val SNP: relevance to human diseases and interaction with environmental factors," Free Radical Research, vol. 47, no. 10, pp. 781-792, 2013.

[18] A. Crawford, R. G. Fassett, D. P. Geraghty et al., "Relationships between single nucleotide polymorphisms of antioxidant enzymes and disease," Gene, vol. 501, no. 2, pp. 89-103, 2012.

[19] S. Shimoda-Matsubayashi, H. Matsumine, T. Kobayashi, Y. Nakagawa-Hattori, Y. Shimizu, and Y. Mizuno, "Structural dimorphism in the mitochondrial targeting sequence in the human manganese superoxide dismutase gene: a predictive evidence for conformational change to influence mitochondrial transport and a Study of Allelic Association in Parkinson's Disease," Biochemical and Biophysical Research Communications, vol. 2, pp. 561-565, 1996.

[20] A. Sutton, A. Imbert, A. Igoudjil et al., "The manganese superoxide dismutase Ala16Val dimorphism modulates both mitochondrial import and mRNA stability," Pharmacogenetics and Genomics, vol. 15, no. 5, pp. 311-319, 2005.

[21] M. A. E. Montano, I. B. M. da Cruz, M. M. M. F. Duarte et al., "Inflammatory cytokines in vitro production are associated with Ala16Val superoxide dismutase gene polymorphism of peripheral blood mononuclear cells," Cytokine, vol. 1, pp. 30-33, 2012.

[22] A. E. Flores, E. T. Pascotini, A. Kegler et al., "ALA16VALMnSOD gene polymorphism and stroke: association with dyslipidemia and glucose levels," Gene, vol. 627, pp. 57-62, 2017.

[23] E. T. Pascotini, A. E. Flores, A. Kegler et al., "Brain-derived neurotrophic factor levels are lower in chronic stroke patients: a relation with manganese-dependent superoxide dismutase ALA16VAL single nucleotide polymorphism through tumor necrosis factor- $\alpha$ and caspases pathways," Journal of Stroke and Cerebrovascular Diseases, vol. 11, pp. 3020-3029, 2018.

[24] P. Wang, Y. Zhu, S. Xi, S. Li, and Y. Zhang, "Association between $M n S O D$ Val16Ala polymorphism and cancer risk: evidence from 33,098 Cases and 37,831 controls," Disease Markers, vol. 2018, Article ID 3061974, 16 pages, 2018.

[25] A. Kegler, A. S. Cardoso, A. L. F. Caprara et al., "Involvement of MnSOD Ala16Val polymorphism in epilepsy: a relationship with seizure type, inflammation, and metabolic syndrome," Gene, vol. 711, article 143924, 2019.

[26] A. T. Berg, S. F. Berkovic, M. J. Brodie et al., "Revised terminology and concepts for organization of seizures and epilepsies: report of the ILAE commission on classification and terminology, 2005-2009," Epilepsia, vol. 51, no. 4, pp. 676-685, 2010.
[27] J. A. Cramer and J. French, "Quantitative assessment of seizure severity for clinical trials: a review of approaches to seizure components," Epilepsia, vol. 42, no. 1, pp. 119-129, 2001.

[28] A. Z. Reznick and L. Packer, "[38] Oxidative damage to proteins: Spectrophotometric method for carbonyl assay," Methods in Enzymology, vol. 233, pp. 357-363, 1994.

[29] D. R. Spitz and L. W. Oberley, "An assay for superoxide dismutase activity in mammalian tissue homogenates," Analytical Biochemistry, vol. 179, no. 1, pp. 8-18, 1989.

[30] S.-D. Chen, A. YW Chang, and Y. C. Chuang, "The potential role of mitochondrial dysfunction in seizure-associated cell death in the hippocampus and epileptogenesis," Journal of Bioenergetics and Biomembranes, vol. 42, no. 6, pp. 461-465, 2010.

[31] B. Menon, K. Ramalingam, and R. V. Kumar, "Oxidative stress in patients with epilepsy is independent of antiepileptic drugs," Seizure, vol. 10, pp. 780-784, 2012.

[32] C. Ikonomidou and A. M. Kaindl, "Neuronal death and oxidative stress in the developing brain," Antioxidants \& Redox Signaling, vol. 14, no. 8, pp. 1535-1550, 2011.

[33] M. Patel and Q. Y. Li, "Age dependence of seizure-induced oxidative stress," Neuroscience, vol. 118, no. 2, pp. 431-437, 2003.

[34] M. Patel, "Mitochondrial dysfunction and oxidative stress: cause and consequence of epileptic seizures," Free Radical Biology \& Medicine, vol. 12, pp. 1951-1962, 2004.

[35] K. Sudha, A. V. Rao, and A. Rao, "Oxidative stress and antioxidants in epilepsy," Clinica Chimica Acta, vol. 303, pp. 19-24, 2001.

[36] V. Cavallucci and M. D'Amelio, "Matter of life and death: the pharmacological approaches targeting apoptosis in brain diseases," Current Pharmaceutical Design, vol. 17, no. 3, pp. 215-229, 2011.

[37] X. Liu, H. Zou, C. Slaughter, and X. Wang, "DFF, a heterodimeric protein that functions downstream of caspase- 3 to trigger DNA fragmentation during apoptosis," Cell, vol. 89, no. 2, pp. 175-184, 1997.

[38] J. Peltola, M. Hurme, A. Miettinen, and T. Keränen, “Elevated levels of interleukin-6 may occur in cerebrospinal fluid from patients with recent epileptic seizures," Epilepsy Research, vol. 2, pp. 129-133, 1998.

[39] D. C. Henshall, R. S. B. Clark, P. D. Adelson, M. Chen, S. C. Watkins, and R. P. Simon, "Alterations in bcl-2 and caspase gene family protein expression in human temporal lobe epilepsy," Neurology, vol. 2, pp. 250-257, 2000.

[40] L. Lorente, M. M. Martín, M. Argueso et al., "Serum caspase-3 levels and mortality are associated in patients with severe traumatic brain injury," BMC Neurology, vol. 15, no. 1, article 228, 2015.

[41] L. Galluzzi, E. Morselli, O. Kepp, and G. Kroemer, "Targeting post-mitochondrial effectors of apoptosis for neuroprotection," Biochimica et Biophysica Acta (BBA) - Bioenergetics, vol. 1787, no. 5, pp. 402-413, 2009.

[42] L. Shi, R. J. Chen, H. Zhang, C. M. Jiang, and J. Gong, "Cerebrospinal fluid neuron specific enolase, interleukin- $1 \beta$ and erythropoietin concentrations in children after seizures," Child's Nervous System, vol. 33, no. 5, pp. 805-811, 2017.

[43] F. L. van de Veerdonk and M. G. Netea, "Toll-like receptors and inflammasomes," in The Inflammasomes, Progress in Inflammation Research, I. Couillin, V. Pétrilli, and F. Martinon, Eds., pp. 123-132, Springer, Basel, 2011. 
[44] F. G. Woermann, S. M. Sisodiya, S. L. Free, and J. S. Duncan, "Quantitative MRI in patients with idiophatic generalized epilepsy. Evidence of widespread cerebral structural changes," Brain, vol. 121, no. 9, pp. 1661-1667, 1998.

[45] A. Sutton, H. Khoury, C. Prip-Buus, C. Cepanec, D. Pessayre, and F. Degoul, "The Ala ${ }^{16}$ Val genetic dimorphism modulates the import of human manganese superoxide dismutase into rat liver mitochondria," Pharmacogenetics, vol. 13, no. 3, pp. 145-157, 2003.

[46] M. V. Clément, A. Ponton, and S. Pervaiz, “Apoptosis induced by hydrogen peroxide is mediated by decreased superoxide anion concentration and reduction of intracellular milieu," FEBS Letters, vol. 440, no. 1-2, pp. 13-18, 1998.

[47] N. Ogusu, J. Saruwatari, H. Nakashima et al., "Impact of the superoxide dismutase 2 Val16Ala polymorphism on the relationship between valproic acid exposure and elevation of $\gamma$-glutamyltransferase in patients with epilepsy: a population pharmacokinetic-pharmacodynamic analysis," PLoS One, vol. 9, no. 11, article e111066, 2014.

[48] W. Löscher, U. Klotz, F. Zimprich, and D. Schmidt, "The clinical impact of pharmacogenetics on the treatment of epilepsy," Epilepsia, vol. 50, no. 1, pp. 1-23, 2009.

[49] M. Ercegovac, N. Jovic, D. Sokic et al., "GSTA1, GSTM1, GSTP1 and GSTT1 polymorphisms in progressive myoclonus epilepsy: a Serbian case-control study," Seizure, vol. 32, pp. 30-36, 2015.

[50] M. R. Ashrafi, S. A. Hosseini, G. R. Zamani et al., "The efficacy of the ketogenic diet in infants and young children with refractory epilepsies using a formula-based powder," Acta Neurologica Belgica, vol. 117, no. 1, pp. 175-182, 2017.

[51] M. J. Brodie, "Diagnosing and predicting refractory epilepsy," Acta Neurologica Scandinavica, vol. 112, no. s181, pp. 36-39, 2005.

[52] J. A. French, "Refractory epilepsy: clinical overview," Epilepsia, vol. 48, no. s1, pp. 3-7, 2007.

[53] J. Arend, A. Kegler, A. L. F. Caprara et al., "Depressive, inflammatory, and metabolic factors associated with cognitive impairment in patients with epilepsy," Epilepsy \& Behavior, vol. 86, pp. 49-57, 2018. 